\title{
Prediction of Outcome in Acute Pancreatitis by the qSOFA and the New ERAP Score
}

\author{
Sebastian Rasch ${ }^{1}$ (1) - Eva-Maria PichImeier ${ }^{1}$. Veit Phillip ${ }^{1}$. Ulrich Mayr ${ }^{1} \cdot$ Roland M. Schmid $^{1} \cdot$ Wolfgang Huber $^{1}$. \\ Tobias Lahmer ${ }^{1}$
}

Received: 5 January 2021 / Accepted: 6 March 2021 / Published online: 26 March 2021

(c) The Author(s) 2021

\begin{abstract}
Background Early identification of patients with acute severe pancreatitis is important for prompt and adequate treatment. Existing scores for pancreatitis are often laborious or require serial patient evaluation, whereas the qSOFA score, that was established to predict outcome in patients with suspected infection, is simple to perform.

Aims and Methods In this cohort study, we analyse the potential of the qSOFA score to predict outcome of patients with acute pancreatitis and refine the qSOFA score by rapid available laboratory parameters to the emergency room assessment of acute pancreatitis (ERAP) score. Validation was performed in a separate patient cohort.

Results In total 203 patients with acute pancreatitis were recruited. The qSOFA score has the potential to predict ICU admission $(\mathrm{AUC}=0.730, p=0.002)$ and organ failure $(\mathrm{AUC}=0.799, p=0.013)$ in acute pancreatitis. Respiratory rate, mental status, blood urea nitrogen and C-reactive protein are the rapid available parameters with the highest individual impact in binary logistic regression analyses. Their combination to the ERAP score can predict severity of acute pancreatitis according to the revised Atlanta classification ( $\mathrm{AUC}=0.689 \pm 0.041, p<0.001$ ), $\mathrm{ICU}$ admission ( $\mathrm{AUC}=0.789 \pm 0.067, p<0.001$ ), multi-organ dysfunction syndrome ( $\mathrm{AUC}=0.963 \pm 0.024, p<0.001$ ) and mortality ( $\mathrm{AUC}=0.952 \pm 0.028, p=0.001)$. The performance and prognostic validity for organ failure and mortality were validated in an independent patient cohort.

Conclusion The qSOFA is a rapidly available prognostic score in acute pancreatitis with limited prognostic validity. A combination with the laboratory parameters BUN and CRP results in the new ERAP score with outstanding prognostic validity for multi-organ dysfunction syndrome and mortality.
\end{abstract}

Keywords Acute pancreatitis $\cdot$ Organ failure $\cdot$ Mortality $\cdot$ Prognostic score $\cdot$ Early assessment

Wolfgang Huber: Unfortunately suddenly deceased during the final drafting of the manuscript.

Sebastian Rasch

sebastian.rasch@tum.de

Eva-Maria Pichlmeier

Eva.Pichlmeier89@web.de

Veit Phillip

Veit.Phillip@mri.tum.de

Ulrich Mayr

Ulrich.Mayr@mri.tum.de

Roland M. Schmid

RolandM.Schmid@mri.tum.de

Tobias Lahmer

Tobias.Lahmer@mri.tum.de

1 Klinik und Poliklinik für Innere Medizin II, Klinikum Rechts der Isar, Technische Universität München, Ismaninger Straße 22, 81675 München, Germany

\section{Introduction}

With an incidence of 30-45/100.000 person years and a mortality of up to $5 \%$, acute pancreatitis is a frequent and potentially lethal disease [1-4]. While the majority of patients have a mild course of the disease and recover within a few days, some patients develop a severe pancreatitis with critical local and systemic complications [5]. Patients can suffer a fulminant systemic inflammatory response syndrome (SIRS) with multiple organ dysfunction syndrome (MODS) or an acute respiratory distress syndrome (ARDS) in the early phase of acute pancreatitis [6]. Therefore, early identification of patients at risk is crucial for adequate management.

Computer-based algorithms of a plethora of risk factors including novel biomarkers and genetic information might soon enable us to calculate each patient's individual risk to 
suffer complications of acute pancreatitis during the course of the disease. But without all this information, initial patient evaluation has to rely on a few quickly available parameters.

For risk stratification, several pancreatitis specific or general scores like the Ranson- and the BISAP score or the APACHE II score have been established [1, 7, 8]. However, all these scores are of limited use in clinical routine as several parameters and/or longitudinal clinical re-evaluation are required. To estimate the risk of patients to suffer multiple organ failure, the sequential organ failure assessment (SOFA) score has recently been developed and is by now the defining criterion of sepsis. In addition, the quick SOFA score (qSOFA) including respiratory rate, systolic blood pressure and altered mentation was validated for rapid preclinical patient evaluation or the emergency room setting and recommended in the sepsis-3 guideline [9-11]. In contrast to the SOFA score, the qSOFA score has not yet been demonstrated to be a reliable predictor of mortality in patients with acute pancreatitis $[12,13]$. However, given its extraordinary simplicity, the qSOFA score would be an appropriate score particularly for the initial patient evaluation in the emergency. Since the qSOFA score is part of the sepsis guideline, it is routinely calculated for internal patients presenting to our emergency room.

Primary objective of this study is to analyse the potential of the qSOFA score to predict severity, need for intensive care treatment, development of MODS and mortality in patients with acute pancreatitis.

Secondary objective is to compare the prognostic potential of qSOFA to established scores including Ranson-, BISAP- and SOFA score as well as laboratory markers including C-reactive protein (CRP), blood urea nitrogen (BUN) and hematocrit.

Tertiary objective is to evaluate if the combination of the qSOFA score with established laboratory markers can improve the prognostic validity and, if applicable, to validate this new prognostic tool in a retrospective patient cohort.

\section{Methods}

For this cohort study, patients presenting with acute pancreatitis to the emergency department at the tertiary referral center Klinikum rechts der Isar der Technischen Universität München from June 2018 to February 2020 were enrolled. Exclusion criteria were as follows: symptom onset $>72 \mathrm{~h}$ before admission, analgosedation, intubation or vasopressor therapy at admission or referral, age $<18$ years and pregnancy. For the diagnosis of acute pancreatitis, two of the following criteria were required: Elevated serum lipase activity above 3 times of the upper limit of the reference range, typical clinical presentation including acute onset of epigastric pain and abdominal tenderness, typical imaging findings in ultrasound or computed tomography. Acute pancreatitis was classified according to the revised Atlanta classification [6]. The study protocol conforms to the ethical guidelines of the 1975 Declaration of HELSINKI as reflected in a prior approval by the institution's human research committee (Ethikkommission der Fakultät für Medizin der Technischen Universität München, project number 216/18 S) on June 12, 2018. Due to the strictly observational design of the study written, informed consent was waived. The study was registered at the German Clinical Trial Registry (No. DRKS00023141). All relevant data are included in the article or provided as supplementary tables.

Four endpoints were analyzed as outcome parameters: severity of acute pancreatitis according to the revised Atlanta classification, admission to intensive care unit (ICU), MODS and in-hospital mortality. Admission to ICU was a clinical decision depending on the condition of the patient. The qSOFA score was compared to the established prognostic tools Ranson score, BISAP score, the sepsis defining SOFA score and the rapidly and routinely available laboratory prognostic parameters C-reactive protein (CRP), blood urea nitrogen (BUN) and hematocrit. Cut-offs for CRP (15 mg/dl), BUN (8.9 mmol/l) and hematocrit (44\%) were used as previously defined by Pongprasobchai et al., the BISAP score and Brown et al. [9-11, 14-17] The scores were obtained at admission and-if necessary-completed within $48 \mathrm{~h}$.

A binary logistic regression model was used to evaluate the independent effect of the individual qSOFA parameters and univariate significant laboratory prognostic parameters on the course and outcome of acute pancreatitis. Factors with a high independent prognostic impact were combined and make up the new emergency room assessment of acute pancreatitis (ERAP) score. The prognostic accuracy of the combined parameters was tested retrospectively in a previous patient cohort. This patient cohort consists of 223 patients who presented to our emergency department with acute pancreatitis between November 2009 and January 2016.

Statistical analysis was performed using IBM SPSS Statistics 25 (SPSS Inc, Chicago, Illinois, USA). Samples were checked for normal distribution using the Shapiro-Wilk test. Descriptive data of normally distributed parameters are presented as mean \pm standard deviation and as median and range for non-parametric parameters. The Mann-Whitney-U and Kruskas-Wallis tests were used to analyse non-parametric variables and the t-test and a one-way analysis of variances (ANOVA) to analyse variables with normal distribution. To compare qualitative parameters, chi-square test and in small samples (expected frequency of test variable less than 5) Fisher's exact test was used. All statistical tests were two-sided with a level of significance ( $p$-value) of 5\%. Receiver operating characteristics (ROC) curve analysis was used to compare 
different prognostic scores and laboratory parameters. The scores and parameters were classified according to their area under the curve (AUC) value in acceptable (AUC 0.7-0.79), excellent (AUC 0.8-0.89) and outstanding (AUC 0.9-1) prognostic tools [18]. To control the false discovery rate due to multiple testing, $\mathrm{p}$ was adjusted (adj. $p$ ) by the Benjamini Hochberg procedure if necessary. To improve the predictive validity of the qSOFA score established, laboratory markers to predict severity of acute pancreatitis were evaluated in our patient cohort. The qSOFA parameters and laboratory markers with $p<0.05$ in univariate analysis were analyzed in a binary logistic regression model with 'enter' as variable selection method.

Table 1 Patients characteristics

\begin{tabular}{ll}
\hline Age & $56.1 \pm 17.3$ \\
Gender (ð̋: †) & $1.3: 1$ \\
Etiology & $n(\%)$ \\
Alcoholic & $97 / 203(47.8 \%)$ \\
Biliary & $62 / 203(30.5 \%)$ \\
Idiopathic & $24 / 203(11.8 \%)$ \\
Hypertriglyceridemia & $11 / 203(5.4 \%)$ \\
Drug induced & $7 / 203(3.4 \%)$ \\
Autoimmune & $2 / 203(1 \%)$ \\
History of chronic pancreatitis & $66 / 203(32.5 \%)$ \\
Atlanta classification & \\
Mild & $130 / 203(64 \%)$ \\
Moderately severe & $59 / 203(29 \%)$ \\
Severe & $14 / 203(6.9 \%)$ \\
Admission to ICU & $14 / 203(6.9 \%)$ \\
Median days on ICU (range) & $8.5(1-76)$ \\
multi-organ dysfunction syndrome $>48 \mathrm{~h}$ & $5 / 203(2.5 \%)$ \\
Necrotizing pancreatitis & $17 / 203(8.4 \%)$ \\
Mortality & $5 / 203(2.5 \%)$ \\
\hline
\end{tabular}

\section{Results}

In total, 203 patients with acute pancreatitis were included in the study. Patient characteristics are reported in Table 1.

CRP and BUN are distributed differently between mild versus moderately severe and severe pancreatitis according to the revised Atlanta classification. Also, Ranson, BISAP, SOFA and qSOFA score significantly differ between these two groups. Although the median qSOFA score is the same, the distribution is significantly different and moderately severe, and severe cases of acute pancreatitis are associated with higher qSOFA values. Details about prognostic markers and scores concerning the revised Atlanta classification are reported in Table 2.

\section{qSOFA}

Of the 203 patients with acute pancreatitis, 179 have a qSOFA score of 0,21 have a qSOFA score of 1 and 3 a score of 2. No patient has a qSOFA score of 3 . The qSOFA score is statistically significant in predicting ICU admission and MODS. The best sensitivity and specificity is achieved with a cut-off of $\geq 1$ (sensitivity $53 \%$, specificity $92 \%$ for ICU admission, $67 \%$ and $90 \%$ for MODS, respectively). The qSOFA score has an AUC of 0.73 (ICU admission) and 0.79 (MODS) in patients with acute pancreatitis.

Regarding sensitivity and specificity, most parameters and scores are only acceptable predictors of a moderately severe or severe pancreatitis. Figure 1 displays ROC curve analysis comparing those scores and parameters as predictors of the primary endpoints and Table 3 shows the corresponding AUC values.

The best score/parameters to predict severity of acute pancreatitis according to the revised Atlanta classification are the Ranson score, SOFA score and BUN. Yet, they have only acceptable AUC values. The best prognostic score for ICU admission in patients with pancreatitis is the Ranson
Table 2 Prognostic parameters /scores (range) and severity of acute pancreatitis

\begin{tabular}{llllr}
\hline Risk parameter/score & All patients & \multicolumn{2}{l}{ Revised Atlanta classification } & \multirow{2}{*}{$\begin{array}{l}\text { Moderately severe } \\
\text { and severe }\end{array}$} \\
\cline { 3 - 4 } & & Mild & $40.8(19.7-63.5)$ & 0.692 \\
\hline Hematocrit $(\%)$ & $40.8(19.7-63.5)$ & $40.8(23.1-47.0)$ & $3.0(0.1-34.6)$ & $\mathbf{0 . 0 0 1}$ \\
CRP (mg/dL) & $1.5(0.1-34.6)$ & $1.4(0.1-30.3)$ & $16(4-68)$ & $<\mathbf{0 . 0 0 1}$ \\
BUN (mg/dL) & $15(4-58)$ & $12(7-24)$ & $3(0-7)$ & $<\mathbf{0 . 0 0 1}$ \\
Ranson & $2(0-7)$ & $1(0-5)$ & $1(0-4)$ & $<\mathbf{0 . 0 0 1}$ \\
BISAP & $1(0-4)$ & $0(0-4)$ & $2(0-16)$ & $<\mathbf{0 . 0 0 1}$ \\
SOFA & $0(0-16)$ & $0(0-6)$ & $0(0-2)$ & $\mathbf{0 . 0 0 4}$ \\
qSOFA & $0(0-2)$ & $0(0-2)$ & & \\
\hline
\end{tabular}

Results in bold are significant with $p<0.05$

$C R P$ C-reactive protein; $B U N$ blood urea nitrogen 
Fig. 1 Receiver operating characteristic (ROC) curve analysis of Ranson, BISAP, SOFA, qSOFA and ERAP score as well as C-reactive protein (CRP), blood urea nitrogen (BUN) and hematocrit
(A) Atlanta classification (mild vs. moderately severe and severe)
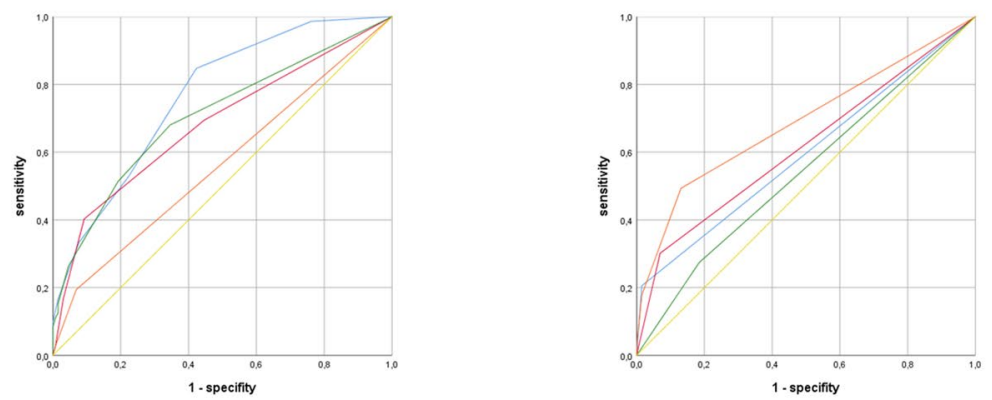

(B) ICU admission
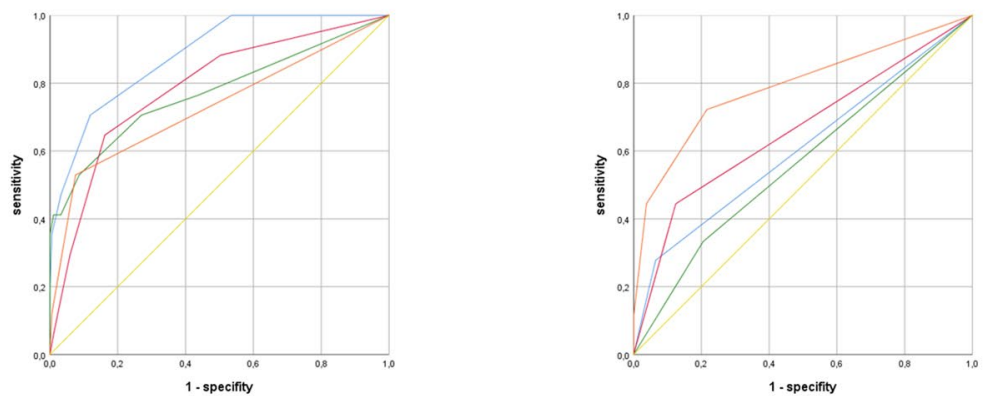

(C) Multi-organ dysfunction syndrome
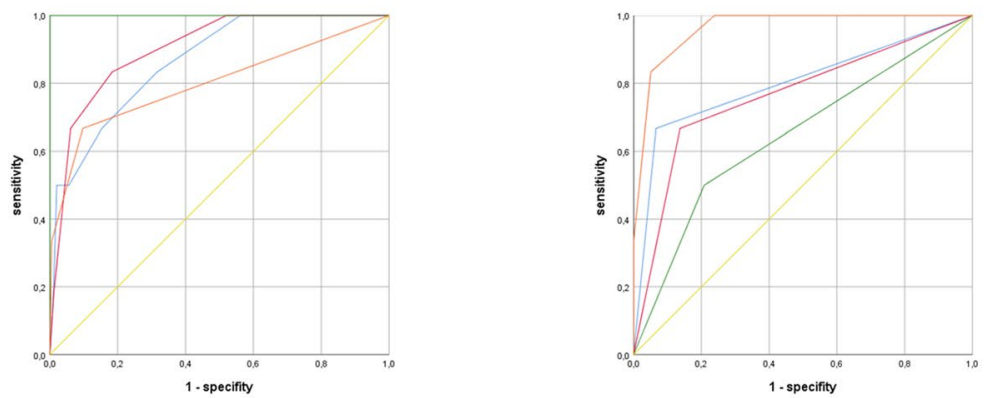

\section{(D) Mortality}
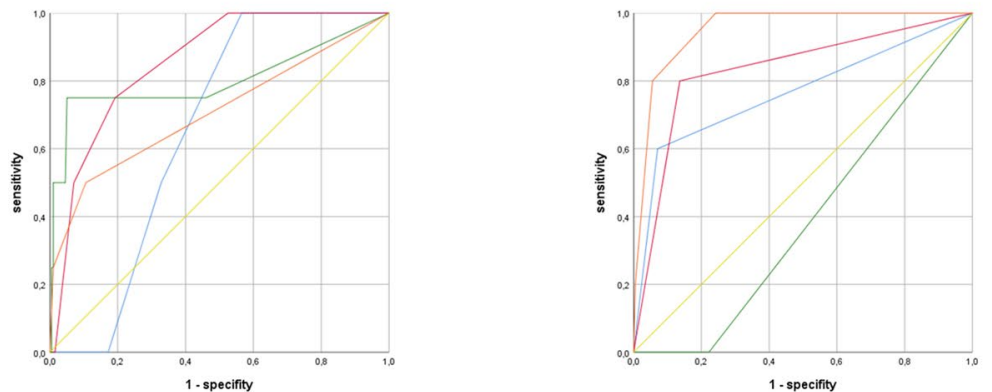

legend

RANSON score
BISAP score
SOFA score
qSOFA score
reference line

legend

blood urea nitrogen C-reactive protein hematocrit ERAP score reference line 
Table 3 AUC values of prognostic scores and parameters (AUC: area under the curve)

\begin{tabular}{lcllll}
\hline Score & AUC & $\begin{array}{l}\text { Standard } \\
\text { deviation }\end{array}$ & $\begin{array}{l}\text { 95\% confidence } \\
\text { interval }\end{array}$ & $p$ (adj.p $=0.032)$ \\
& & & & \\
\hline Atlanta classification & & & & \\
Ranson & 0.765 & 0.033 & 0.699 & 0.831 & $<\mathbf{0 . 0 0 0}$ \\
BISAP & 0.683 & 0.041 & 0.603 & 0.763 & $\mathbf{0 . 0 0 0}$ \\
SOFA & 0.706 & 0.040 & 0.628 & 0.784 & $\mathbf{0 . 0 0 0}$ \\
qSOFA & 0.563 & 0.043 & 0.478 & 0.648 & 0.139 \\
BUN & 0.595 & 0.043 & 0.510 & 0.680 & $\mathbf{0 . 0 2 5}$ \\
CRP & 0.616 & 0.043 & 0.532 & 0.700 & $\mathbf{0 . 0 0 6}$ \\
Hematocrit & 0.545 & 0.043 & 0.461 & 0.628 & 0.291 \\
ICU admission & & & & & \\
Ranson & $\mathbf{0 . 8 8 3}$ & 0.039 & 0.806 & 0.960 & $\mathbf{0 . 0 0 0}$ \\
BISAP & 0.786 & 0.060 & 0.668 & 0.903 & $\mathbf{0 . 0 0 0}$ \\
SOFA & 0.771 & 0.074 & 0.627 & 0.915 & $\mathbf{0 . 0 0 0}$ \\
qSOFA & 0.730 & 0.077 & 0.579 & 0.881 & $\mathbf{0 . 0 0 2}$ \\
BUN & 0.606 & 0.078 & 0.454 & 0.759 & 0.136 \\
CRP & 0.660 & 0.076 & 0.512 & 0.808 & $\mathbf{0 . 0 2 5}$ \\
Hematocrit & 0.564 & 0.074 & 0.418 & 0.710 & 0.371 \\
Multi-organ & dysfunction syndrom & & & \\
Ranson & $\mathbf{0 . 8 6 4}$ & 0.068 & 0.732 & 0.997 & $\mathbf{0 . 0 0 2}$ \\
BISAP & $\mathbf{0 . 9 0 2}$ & 0.054 & 0.797 & 1.000 & $\mathbf{0 . 0 0 1}$ \\
SOFA & $\mathbf{1 . 0 0 0}$ & 0.000 & 1.000 & 1.000 & $\mathbf{0 . 0 0 0}$ \\
qSOFA & 0.799 & 0.119 & 0.566 & 1.000 & $\mathbf{0 . 0 1 3}$ \\
BUN & $\mathbf{0 . 8 0 0}$ & 0.116 & 0.572 & 1.000 & $\mathbf{0 . 0 1 2}$ \\
CRP & 0.765 & 0.115 & 0.540 & 0.990 & $\mathbf{0 . 0 2 7}$ \\
Hematocrit & 0.646 & 0.124 & 0.402 & 0.890 & 0.224 \\
Mortality & & & & & \\
Ranson & 0.652 & 0.064 & 0.525 & 0.778 & 0.300 \\
BISAP & $\mathbf{0 . 8 5 6}$ & 0.072 & 0.715 & 0.997 & $\mathbf{0 . 0 1 5}$ \\
SOFA & $\mathbf{0 . 8 0 2}$ & 0.159 & 0.490 & 1.000 & 0.039 \\
qSOFA & 0.708 & 0.160 & 0.393 & 1.000 & 0.155 \\
BUN & 0.765 & 0.134 & 0.502 & 1.000 & 0.043 \\
Hematocrit & $\mathbf{0 . 3 8 9}$ & 0.103 & 0.186 & 0.592 & 0.396 \\
\hline & & & & &
\end{tabular}

Results in bold are significant with $p<0.05$ score with an excellent AUC. Due to similar definitions, the SOFA score very accurately predicts patients who are at risk to develop a MODS. According to the AUC, the BISAP score is an outstanding tool and the Ranson score as well as BUN are excellent predictors of MODS.

The BISAP- and SOFA scores as well as CRP are excellent predictors of mortality in acute pancreatitis.

\section{Emergency Room Assessment of Acute Pancreatitis (ERAP) Score}

To analyse the individual qSOFA parameters for their independent prognostic power, the endpoint MODS is used as the qSOFA score has its best AUC for the prediction of MODS in acute pancreatitis. In univariate analysis, BUN above $8.9 \mathrm{mmol} / 1(66.7 \%$ vs. $6.6 \%, p<0.001)$ and CRP above $15 \mathrm{mg} / \mathrm{dl}(66.7 \%$ vs. $13.7 \%, p=0.005)$ are significantly associated with MODS as opposed to hematocrit above $44 \%$ (50\% vs. $20.8 \%, p=0.117$ ). In a binary logistic regression model with MODS as dependent variable and respiratory rate $>21 / \mathrm{min}$, systolic blood pressure $<101 \mathrm{mmHg}$, GCS $<15, \mathrm{BUN}>8.9 \mathrm{mmol} / \mathrm{l}$ and $\mathrm{CRP}>15 \mathrm{mg} / \mathrm{dl}$ as independent variables, all prognostic variables but systolic blood pressure are statistically significant independent prognostic parameters. Details on the regression model are reported in Table 4.

The ERAP score consisting of two clinical parameters (respiratory rate $>21 / \mathrm{min}$ and $\mathrm{GCS}<15$ ) and two laboratory parameters (BUN $>8.9 \mathrm{mmol} / \mathrm{l}$ and CRP $>15 \mathrm{mg} / \mathrm{dl}$ ) can statistically significantly predict severity of acute pancreatitis (AUC $0.689 \pm 0.041, p<0.001$ ), ICU admission (AUC $0.789 \pm 0.067, p<0.001$ ), MODS (AUC 0.963 \pm 0.024 , $p<0.001$ ) and mortality (AUC $0.952 \pm 0.028, p=0.001$ ). Corresponding ROC-curves are displayed in Fig. 1. An ERAP $>1$ predicts MODS with a sensitivity of $83.3 \%$ and a specificity of $94.9 \%$ and mortality with $80 \%$ and $94.4 \%$, respectively.
Table 4 Binary logistic regression model with MODS as dependent variable

\begin{tabular}{|c|c|c|c|c|c|c|}
\hline \multirow{2}{*}{$\begin{array}{l}\text { Variable } \\
\text { BUN>8.9 mmol/1 }\end{array}$} & \multirow{2}{*}{$\begin{array}{l}\begin{array}{l}\text { Regression } \\
\text { coefficient B }\end{array} \\
2.98\end{array}$} & \multirow{2}{*}{$\begin{array}{l}\text { Standard error } \\
1.26\end{array}$} & \multirow{2}{*}{$\begin{array}{l}\begin{array}{l}\text { Adjusted odds } \\
\text { ratio }(\operatorname{expB})\end{array} \\
19.74\end{array}$} & \multicolumn{2}{|c|}{$\begin{array}{l}95 \% \text { CI of adjusted } \\
\text { odds ratio }\end{array}$} & \multirow{2}{*}{$\begin{array}{l}p \\
\mathbf{0 . 0 1 8}\end{array}$} \\
\hline & & & & 1.68 & 231.69 & \\
\hline $\mathrm{AF}>21 / \mathrm{min}$ & 3.81 & 1.38 & 45.14 & 3.01 & 676.94 & 0.006 \\
\hline GCS $<15$ & 3.36 & 1.55 & 28.81 & 1.38 & 603.44 & 0.030 \\
\hline $\begin{array}{l}\text { Syst. blood pres- } \\
\text { sure }<101 \mathrm{mmHg}\end{array}$ & 0.77 & 2.95 & 2.16 & 0.01 & 695.05 & 0.794 \\
\hline $\mathrm{CRP}<15 \mathrm{mg} / \mathrm{dl}$ & 2.79 & 1.34 & 16.33 & 1.19 & 223.97 & 0.037 \\
\hline
\end{tabular}

Results in bold are significant with $p<0.05$

$\mathrm{R}^{2}$ (Nagelkerke) $=0.598 ; p<0.001$ 


\section{ERAP Validation}

To validate the accuracy of the ERAP score in predicting outcome in acute pancreatitis, the score was retrospectively applied on a previous patient cohort of 223. Mean age (54 \pm 18 years) and gender distribution $(\widehat{\delta}:$ ㅇ $1.4: 1)$ are comparable to our study cohort. Patient characteristics of the validation cohort are displayed in supplementary Table 1 and AUC values for the prediction of ICU admission, MODS, and mortality in Table 5.

\section{Discussion}

Acute pancreatitis is the third most common diagnosis at discharge of hospitalized patients in gastroenterology and hepatology in the United States [19]. As only few of those patients develop a severe pancreatitis but patients with severe pancreatitis have a mortality of up to $30 \%$ it is important to early identify patients at risk of a severe pancreatitis [20, 21]. For this reason, prognostic scores and markers were evaluated and established in recent decades. An ideal prognostic marker is quick and easy to obtain and widely available at hospital admission of the patient. Established laboratory markers like CRP are well validated and can accurately predict outcome in acute pancreatitis. However, previous studies evaluating CRP as predictor in acute pancreatitis showed the best results for CRP $24 \mathrm{~h}$ after admission in contrast to markedly lower negative and positive predictive values at the time of admission [1]. Sepsis with hyper-inflammation resulting in hypovolemic shock shares similar pathogenic features with acute pancreatitis. Also, early recognition and adequate treatment is essential for the outcome in both diseases [22]. The qSOFA score is quick and easy to obtain and has an excellent predictive validity for mortality in sepsis, particularly outside of an ICU, i.e., out-of-hospital, in the emergency department, or on a general hospital ward [9]. So far, the qSOFA score has not been associated with a complicated course of acute pancreatitis [23]. According to our data, the qSOFA score can predict ICU admission and MODS in acute pancreatitis. There is also an association of the qSOFA score to the severity of pancreatitis although the predictive validity is not significant in ROC curve analysis. However, the prognostic validity of the qSOFA score according to its AUC values is limited and particularly the Ranson- and BISAP scores, that were developed and validated for acute pancreatitis, have better AUC values. However, the BISAP score consists of 8 different parameters and especially the Ranson score requires repeated assessment of clinical and laboratory parameters. Thus, to get a quick but reliable prognostic tool, we modified the qSOFA score and identified two clinical and two laboratory parameters with high prognostic impact. Altered mentation and respiratory rate as clinical parameters of the qSOFA score and CRP and BUN as quickly and routinely available laboratory parameters were selected. We named the combination of these parameters 'Emergency Room Assessment of acute Pancreatitis'-ERAP score. Similar to the BISAP score, the ERAP score can be calculated with routinely available clinical and laboratory parameters at initial patient presentation. However, by reducing the required parameters from 8 to only 4 , the ERAP score provides an extraordinarily feasible screening tool.

The ERAP score has outstanding AUC values for predicting MODS and mortality and an acceptable AUC for ICU admission. Only the Ranson score, which is laborious to calculate, is superior in predicting ICU admission. In general, AUC values of all prognostic parameters are lowest for the prediction of severity. This can be explained by the fact, that some of the scores were established before publication of the revised Atlanta classification in 2012. In addition, the revised Atlanta classification defines the severity of pancreatitis by local as well as systemic complications that can occur early and late in the course of the disease like walled off necrosis. Such heterogeneous complications in pathogenetically different states of the disease are by nature hard to predict by selected parameters.

The ERAP score was validated in an existing patient cohort that is comparable to the study cohort and sensitivity, and specificity rates did not differ between the validation cohort and the study cohort. This confirms the potential of the ERAP score as quick and easy score to assess the risk of
Table 5 Validation of the ERAP score in predicting the outcome of acute pancreatitis

\begin{tabular}{lllllllll}
\hline & $n$ & AUC & SD & $p$ & $95 \%$ CI & & PPV (\%) & NPV (\%) \\
\hline $\begin{array}{l}\text { ICU admission } \\
\begin{array}{l}\text { Multiorgan } \\
\text { dysfunction }\end{array}\end{array}$ & $47 / 223$ & $\mathbf{0 . 7 8 7}$ & 0.042 & 0.000 & 0.703 & 0.870 & $\mathbf{7 3 . 1}$ & $\mathbf{8 5 . 8}$ \\
$\begin{array}{l}\text { syndrome } \\
\text { Mortality }\end{array}$ & $14 / 223$ & $\mathbf{0 . 9 2 2}$ & 0.025 & 0.000 & 0.872 & 0.971 & $\mathbf{3 4 . 6}$ & $\mathbf{9 7 . 5}$ \\
\hline
\end{tabular}

Results in bold are significant with $p<0.05$

$A U C$ area under the curve, $S D$ standard deviation, $C I$ confidence interval, $P P V$ positive predictive value, $N P V$ negative predictive value 
organ failure and mortality in patients with acute pancreatitis within the first hour at the emergency department.

\section{Limitations of the Study}

Although the prognostic potential of the qSOFA score and the new ERAP score were analyzed and established with prospective patient data, validation of the ERAP score was performed on an existing patient cohort. Also both cohorts were recruited at a single center which inherits the risk of a referral bias. AUC values of established scores and markers in our patient cohort are comparable to reported data $[7,8]$. With an AUC above 0.8 , sensitivity and specificity of the SOFA score are further in line with recent publications [13]. Contrary to previous reports, the SOFA score is statistically just not significant in predicting mortality after multiple testing adjustment, which might be due to a comparably low mortality rate. However, the statistical power of the data should be interpreted with caution due to the low number of patients at risk for rare end points, like mortality, and its validation in a retrospective patient cohort. Prospective confirmation of the results in a larger multicenter cohort would be desirable.

\section{Conclusion}

The qSOFA is a rapidly available prognostic score with limited prognostic value in acute pancreatitis. Combining the two qSOFA criteria respiratory rate and mental status with the laboratory parameters BUN and CRP results in the emergency room assessment of acute pancreatitis (ERAP) score, which is a sensitive prognostic score for the prediction of MODS and mortality in acute pancreatitis.

Supplementary Information The online version contains supplementary material available at https://doi.org/10.1007/s10620-021-06945-z.

Acknowledgments Tobias Lahmer received travel grants from Gilead, Pfizer and MSD. Sebastian Rasch received travel grants from Gilead. Wolfgang Huber collaborated with Pulsion Medical Systems SE, Feldkirchen, Germany as member of the Medical Advisory Board.

Funding Open Access funding enabled and organized by Projekt DEAL. No external funding was obtained for this study.

\section{Declaration}

Conflict of interest The authors declare that they have no conflict of interest.

Ethical approval All procedures performed in studies involving human participants were in accordance with the ethical standards of the institutional and/or national research committee and with the 1964 Declaration of HELSINKI and its later amendments or comparable ethical standards.
Open Access This article is licensed under a Creative Commons Attribution-NonCommercial 4.0 International License, which permits any non-commercial use, sharing, adaptation, distribution and reproduction in any medium or format, as long as you give appropriate credit to the original author(s) and the source, provide a link to the Creative Commons licence, and indicate if changes were made. The images or other third party material in this article are included in the article's Creative Commons licence, unless indicated otherwise in a credit line to the material. If material is not included in the article's Creative Commons licence and your intended use is not permitted by statutory regulation or exceeds the permitted use, you will need to obtain permission directly from the copyright holder. To view a copy of this licence, visit $\mathrm{http} / / /$ creativecommons.org/licenses/by-nc/4.0/.

\section{References}

1. Phillip V, Steiner JM, Algul H. Early phase of acute pancreatitis: assessment and management. World J Gastrointest Pathophysiol. 2014;5:158-168. https://doi.org/10.4291/wjgp.v5.i3.158.

2. Roberts SE, Akbari A, Thorne K, Atkinson M, Evans PA. The incidence of acute pancreatitis: impact of social deprivation, alcohol consumption, seasonal and demographic factors. Aliment Pharmacol Ther. 2013;38:539-548. https://doi.org/10.1111/apt. 12408.

3. Satoh K, Shimosegawa T, Masamune A, Hirota M, Kikuta K, Kihara Y et al. Nationwide epidemiological survey of acute pancreatitis in Japan. Pancreas. 2011;40:503-507. https://doi.org/10. 1097/MPA.0b013e318214812b.

4. Phillip V, Huber W, Hagemes F, Lorenz S, Matheis U, Preinfalk $S$ et al. Incidence of acute pancreatitis does not increase during Oktoberfest, but is higher than previously described in Germany. Clin Gastroenterol Hepatol. 2011;9:995-1000. https://doi.org/10. 1016/j.cgh.2011.06.016.

5. Whitcomb DC. Clinical practice. Acute pancreatitis. $N$ Engl J Med. 2006;354:2142-2150. https://doi.org/10.1056/NEJMcp0549 58.

6. Banks PA, Bollen TL, Dervenis C, Gooszen HG, Johnson CD, Sarr MG et al. Classification of acute pancreatitis-2012: revision of the Atlanta classification and definitions by international consensus. Gut. 2013;62:102-111. https://doi.org/10.1136/gutjnl2012-302779 (Epub 2012 Oct 25).

7. Juneja D, Gopal PB, Ravula M. Scoring systems in acute pancreatitis: which one to use in intensive care units? J Crit Care. 2010;25:358. https://doi.org/10.1016/j.jcrc.2009.12.010.

8. Vasudevan S, Goswami P, Sonika U, Thakur B, Sreenivas V, Saraya A. Comparison of various scoring systems and biochemical markers in predicting the outcome in acute pancreatitis. Pancreas. 2017;47:65-71. https://doi.org/10.1097/mpa.0000000000 000957.

9. Seymour CW, Liu VX, Iwashyna TJ, Brunkhorst FM, Rea TD, Scherag A et al. Assessment of clinical criteria for sepsis: for the third international consensus definitions for sepsis and septic shock (Sepsis-3). JAMA. 2016;315:762-774. https://doi.org/10. 1001/jama.2016.0288.

10. Shankar-Hari M, Phillips GS, Levy ML, Seymour CW, Liu VX, Deutschman CS et al. Developing a new definition and assessing new clinical criteria for septic shock: for the third international consensus definitions for sepsis and septic shock (Sepsis-3). JAMA. 2016;315:775-787. https://doi.org/10.1001/jama.2016. 0289 .

11. Singer M, Deutschman CS, Seymour CW, Shankar-Hari M, Annane D, Bauer $\mathrm{M}$ et al. The third international consensus definitions for sepsis and septic shock (Sepsis-3). JAMA. 2016;315:801810. https://doi.org/10.1001/jama.2016.0287. 
12. Adam F, Bor C, Uyar M, Demirag K, Cankayali I. Severe acute pancreatitis admitted to intensive care unit: SOFA is superior to Ranson's criteria and APACHE II in determining prognosis. Turk J Gastroenterol Off J Turk Soc Gastroenterol. 2014;24:430-435.

13. Tee YS, Fang HY, Kuo IM, Lin YS, Huang SF, Yu MC. Serial evaluation of the SOFA score is reliable for predicting mortality in acute severe pancreatitis. Medicine 2018;97:e9654. https://doi. org/10.1097/md.0000000000009654.

14. Pongprasobchai S, Jianjaroonwong V, Charatcharoenwitthaya $P$, Komoltri C, Tanwandee T, Leelakusolvong S et al. Erythrocyte sedimentation rate and C-reactive protein for the prediction of severity of acute pancreatitis. Pancreas. 2010;39:1226-1230. https://doi.org/10.1097/MPA.0b013e3181deb33e.

15. Brown A, Orav J, Banks PA. Hemoconcentration is an early marker for organ failure and necrotizing pancreatitis. Pancreas. 2000;20:367-372. https://doi.org/10.1097/00006676-20000 5000-00005.

16. Wu BU, Johannes RS, Sun X, Tabak Y, Conwell DL, Banks PA. The early prediction of mortality in acute pancreatitis: a large population-based study. Gut. 2008;57:1698-1703. https://doi.org/ 10.1136/gut.2008.152702.

17. Ranson JH, Rifkind KM, Roses DF, Fink SD, Eng K, Spencer FC. Prognostic signs and the role of operative management in acute pancreatitis. Surg Gynecol Obstet. 1974;139:69-81.

18. Mandrekar JN. Receiver operating characteristic curve in diagnostic test assessment. J Thorac Oncol. 2010;5:1315-1316. https:// doi.org/10.1097/JTO.0b013e3181ec173d.

19. Peery AF, Crockett SD, Murphy CC, Lund JL, Dellon ES, Williams JL et al. Burden and cost of gastrointestinal, liver, and pancreatic diseases in the united states: update 2018. Gastroenterology. 2019;156:254-272. https://doi.org/10.1053/j.gastro.2018. 08.063 .

20. Lankisch PG, Assmus C, Maisonneuve P, Lowenfels AB. Epidemiology of pancreatic diseases in Luneburg County. A study in a defined german population. Pancreatology. 2002;2:469-477. https://doi.org/10.1159/000064713.

21. Lankisch PG, Apte M, Banks PA. Acute pancreatitis. Lancet. 2015;386:85-96. https://doi.org/10.1016/S0140-6736(14) 60649-8.

22. Christopher WS, Department of Critical Care Medicine UoPSoMPP, Clinical Research I, Systems Modeling of Acute Illness Center PP, Vincent XL, Division of Research KPOC et al. Assessment of clinical criteria for sepsis: for the third international consensus definitions for sepsis and septic shock (Sepsis-3). JAMA. 2018;315:762-774. https://doi.org/10.1001/jama.2016.0288.

23. Hallac A, Puri N, Applebury D, Myers K, Dhumal P, Thatte A et al. The value of quick sepsis-related organ failure assessment scores in patients with acute pancreatitis who present to emergency departments: a three-year cohort study. Gastroenterology Res. 2019;12:67-71. https://doi.org/10.14740/gr1132.

Publisher's Note Springer Nature remains neutral with regard to jurisdictional claims in published maps and institutional affiliations. 